\title{
Mitochondrial replacement by pre-pronuclear transfer in human embryos
}

Cell Research (2017) 27:834-837. doi:10.1038/cr.2017.48; published online 4 April 2017

\section{Dear Editor,}

Mitochondrial DNA (mtDNA) diseases are maternally transmitted to offspring [1]. It has been proposed to prevent mtDNA disease inheritance through mitochondrial replacement therapy (MRT), using nonpathogenic mitochondria from donor oocytes to replace defective maternal mitochondria. Three strategies, including maternal spindle transfer (MST), pronuclear transfer (PNT) and polar body transfer (PBT), have been applied in human embryos [2-5]. All these techniques require the use of cytochalasin $\mathrm{B}(\mathrm{CB})$ or nocodazole, the cytoskeleton disruptors, to assist the isolation of the chromosome-spindle complex or pronucleus. However, the safety of using these cytoskeleton disruptors in clinical applications of human embryo-related manipulation has not been rigorously evaluated. In this study, we show a novel technique, in which female pre-pronucleus (PPN) can be isolated shortly after fertilization in a condition without cytoskeleton disruptors and then be successfully used for MRT, leading to the generation of human embryonic stem cell (ESC) lines with mtDNA carryover at very low levels.

At telophase of the second meiosis of oocytes, the PPN adheres to the extruding second polar body (PB2). In human, PPN forms at 3.5-4 $\mathrm{h}$ after fertilization and will not separate completely from PB2 within $6 \mathrm{~h}$ post fertilization. Thereafter, it will be enclosed by the pronuclear envelope and forms the female pronucleus. We reasoned that if PPN could be easily isolated together with the extruding PB2, the use of $\mathrm{CB}$ or nocodazole can be avoided. In addition, this simple procedure, termed PPN transfer (PPNT), may yield female PPN with minimal mtDNA carryover that can be used as a novel source of female nuclear material for MRT (Figure 1A).

To test the feasibility of PPNT in human zygotes, we used immature MI oocytes donated by patients who were taking in vitro fertilization (IVF) treatments. MI oocytes were kept in in vitro maturation (IVM) medium for 18$24 \mathrm{~h}$. Matured oocytes with the distinct first polar body (PB1) were collected and used for intracytoplasmic sperm injection (ICSI). PB1 was then removed. 3.5-4 hours post ICSI, zygotes with extruding PB2 were collected for isolation of PPN (Figure 1A). The PPN and PB2 complex could be easily isolated from the zygote in medium without $\mathrm{CB}$ using a pipette with a diameter of $20 \mu \mathrm{m}$ (Figure 1B). PPN and PB2 complexes were kept in culture medium of human embryos for 2-3 h; PPN was then separated from PB2 spontaneously (Figure 1C). PPN was briefly exposed in Sendai virus extract and injected into the perivitelline space of a donor zygote whose PB1, PB2 and PPN have been removed through the same method. Membrane fusion between PPN and the donor zygote was usually observed within 20-30 min, resulting in reconstructed embryos.

From a total of 279 MI oocytes, we obtained 211 MII oocytes through IVM. All of them were used for ICSI, resulting in 164 survived zygotes with PB2, among which 80 were used for PPNT and 84 served as non-manipulated ICSI controls. We successfully performed PPNT between 39 ICSI zygotes from different donors (Heterologous PPNT, Group 1). As a sub-control, 37 zygotes were obtained via PPNT performed between different zygotes of a same donor (Homologous, Group 2). Almost all manipulated embryos from the two groups formed two visible pronuclei ( 37 of 39 and 37 of 37, respectively), similar to non-manipulated ICSI controls (83 of 84). After 5-7 days of in vitro culturing, PPNT zygotes and control zygotes developed into blastocysts at a similar efficiency (Figure 1D-1E). Taken together, these results indicate that the PPNT procedure does not affect the preimplantation development of reconstructed embryos.

A total of 10 good-quality blastocysts (4BC or better by Gardner's criteria) were produced through PPNT in Group 1 and were cryopreserved until further analyses. Nine of them were successfully thawed and used for karyotype analysis. DNA samples of trophoblast cells dissociated by trophectoderm (TE) biopsy (Figure 1F) of PPNT and control embryos were used for preimplantation genetic screening (PGS). The results showed that 6 blastocysts in Group 1 were euploid $(6 / 9,66.67 \%$; Supplementary information, Table S1B). This rate is com- 
A

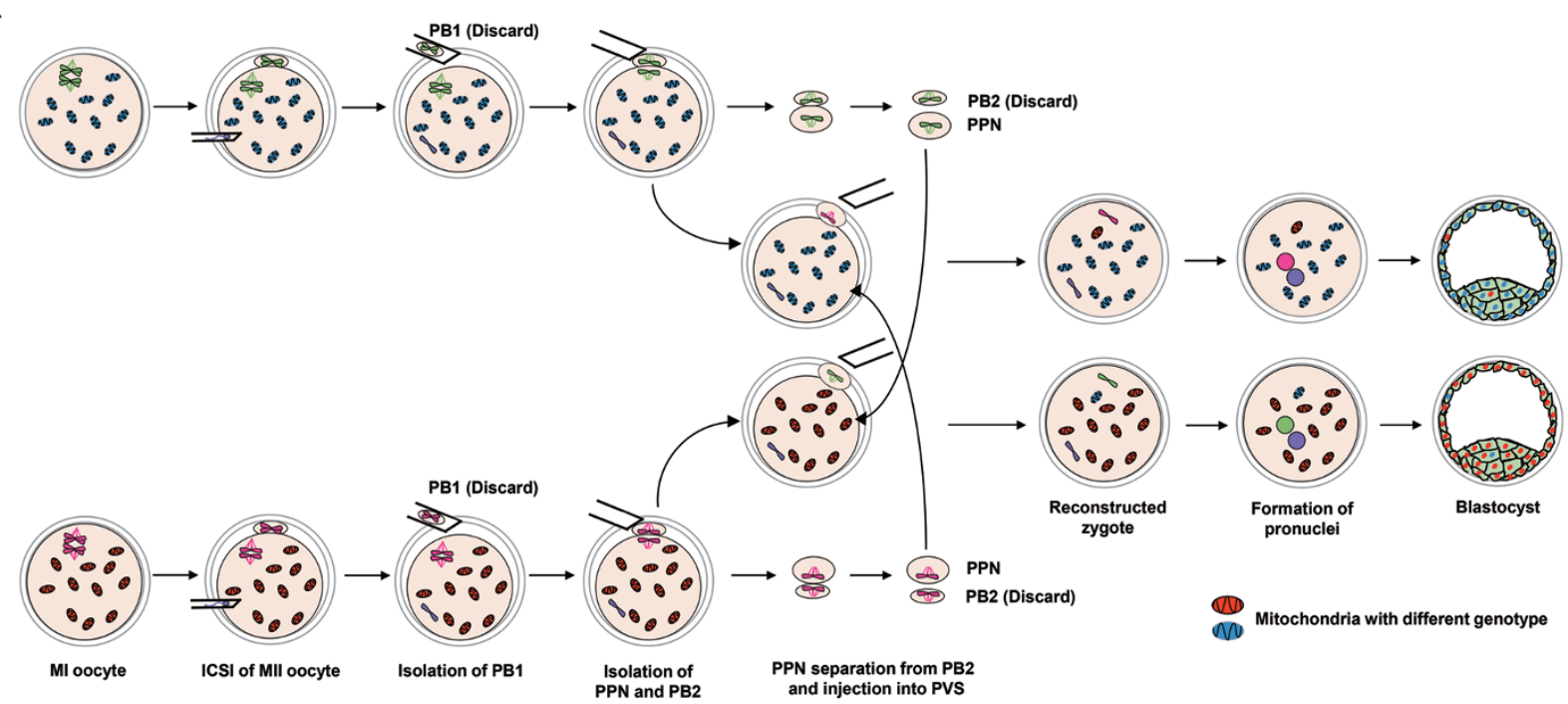

B

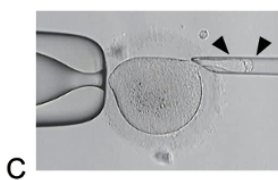

C

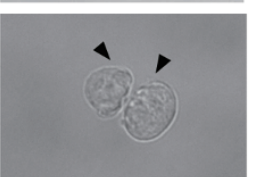

D

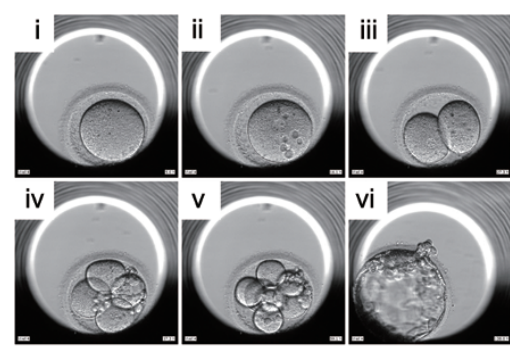

F Trophectoderm

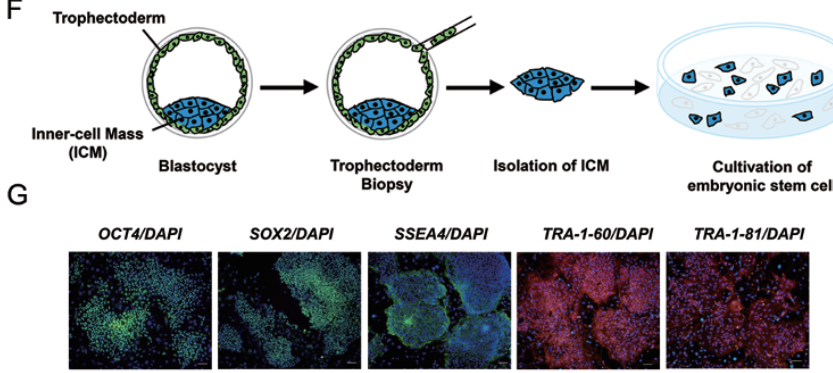

E

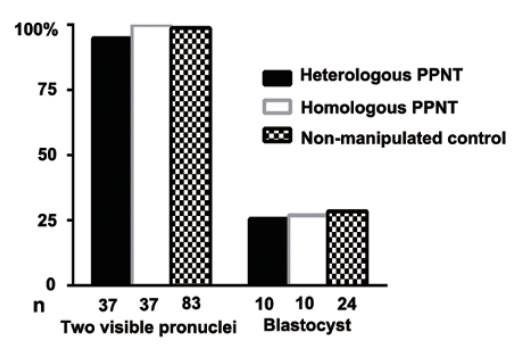

$\mathrm{H}$

\begin{tabular}{cccccccccc}
\hline & & ESC & ESC & ESC & ESC & \multirow{2}{*}{ EB } & \multirow{2}{c}{ Teratoma } & \multicolumn{2}{c}{ Differentiation } \\
\cline { 7 - 9 } & & P5 & P10 & P15 & P20 & & & Cardiomyocytes & Neuron \\
\hline ESC-1 & $0.53 \%$ & $0.58 \%$ & $0.15 \%$ & $0.12 \%$ & $0.11 \%$ & $0.21 \%$ & NT & $0.19 \%$ & $0.11 \%$ \\
ESC-2 & $0.10 \%$ & $0.23 \%$ & $0.02 \%$ & $0.25 \%$ & $0.25 \%$ & $0.11 \%$ & $0.04 \%$ & $0.19 \%$ & $0.20 \%$ \\
ESC-3 & $0.19 \%$ & $0.50 \%$ & $0.37 \%$ & $0.16 \%$ & $0.38 \%$ & $0.14 \%$ & $0.06 \%$ & $0.14 \%$ & $0.13 \%$ \\
\hline
\end{tabular}

Figure 1 Generation of reconstructed embryos through PPNT. (A) Schematic diagram of PPN. (B) Isolation of PPN (left arrow) and PB2 (right arrow). (C) PPN (right arrow) separates from PB2 (left arrow). (D) Development of reconstructed embryos in vitro. (i) Reconstructed zygote; (ii) pronucleus formation; (iii) two-cell stage; (iv) four-cell stage; (v) eight-cell stage; (vi) blastocyst stage. (E) Blastocyst development after heterologous PPNT $(n=39)$, homologous PPNT $(n=37)$ and non-manipulated control ( $n=84)$. (F) Schematic diagram showing TE biopsy and ESC derivation. (G) Expression of human pluripotency markers in ESCs derived from reconstructed blastocysts. Shown are the representative immunostaining images of ESC-1 colonies. Scale bar, $100 \mu \mathrm{m}$. (H) MtDNA carryover in three ESC lines during extended culture and differentiation. NT, not tested.

parable to that of non-manipulated ICSI control group $(15 / 24,62.5 \%)$. Of note, results from a number of IVF centers in the United States indicated that the euploidy rate of blastocyst biopsies of IVF patients under 35 years old is $\sim 60 \%$ [6], suggesting that PPNT per se does not lead to aneuploidy of the reconstructed embryos. Meanwhile, we assessed SNP variants of mtDNA between the donor and recipient to distinguish mtDNA origins. MtD-
NA samples from trophoblast cells were analyzed by droplet digital PCR (ddPCR) [7]. MtDNA carryover of the 9 PPNT blastocysts was at an average level of $1.04 \%$ $(0.10 \%-4.67 \%)$. Interestingly, the 6 euploid blastocysts had a lower level of mtDNA leftover $(0.36 \%, 0.10 \%$ $0.58 \%$; Supplementary information, Table S1B).

Next, we attempted to establish ESC lines from PPNT blastocysts. Inner cell masses (ICMs) from the 6 euploid 
blastocysts were dissociated for ESC derivation (Figure $1 \mathrm{~F})$. Three ESC lines were successfully generated (termed ESC-1, ESC-2, and ESC-3). These cells were cultured in the standard human ESC culture conditions for over 20 passages. PPNT ESCs exhibited typical colony morphology and expressed classical pluripotency markers, including alkaline phosphatase, OCT4, SOX2, SSEA4, TRA-1-60 and TRA-1-81 (Figure 1G and Supplementary information, Figure S1A-S1C). Cytogenetic analyses via G-banding indicated that all three lines exhibited normal karyotype with no detectable chromosomal abnormalities (Supplementary information, Figure S1D).

Having demonstrated the low level of mtDNA carryover in PPNT embryos, we next tested the mtDNA carryover during ESC proliferation and differentiation. DNA samples of ESCs at different passages were collected for ddPCR analysis. The results showed that the ratio of mtDNA carryover was relatively stable during ESC proliferation (Figure $1 \mathrm{H}$ ). We also assessed mtDNA carryover during ESC differentiation. To this end, we analyzed the differentiation potential of ESCs derived from PPNT embryos by in vitro and in vivo assays. First, PPNT ESCs were suspended for the formation of embryoid body (EB) in vitro (Supplementary information, Figure S2A). Immunostaining analysis showed EBs from the three cell lines could differentiate into cells from three germ layers (Supplementary information, Figure S2B). Second, PPNT ESCs could differentiate into cardiomyocytes and neurons in vitro through reported protocols [8] (Supplementary information, Figure S2C). Third, teratomas consisting of tissues from three germ layers were formed after injection of ESC suspensions into immune-deficient mice (Supplementary information, Figure S2D). We collected DNA samples from differentiated cells derived from neurons/cardiomyocytes and teratomas for mtDNA carryover analysis. The results showed that the ratio of mtDNA carryover was low and relatively stable in all tested samples (Figure 1H). Taken together, these results demonstrate that PPNT can be used to efficiently generate blastocysts and ESCs with low levels of mtDNA carryover, showing its potential in clinical application for preventing the inheritance of mtDNA diseases.

MRT can potentially prevent the transmission of mutant mtDNA to next generations. Two strategies, MST and PNT, have been successfully tested in human embryos in vitro. Most recently, the PB1 transfer has been successfully employed in human oocytes for the generation of reconstructed embryos and ESCs [5]. In this study, we first modified the MRT strategy by using PPN for transfer for the following reasons. First, PPN is smaller compared with the spindle-chromosomal complex and pronucleus, and thus has less mitochondria attached. Second, PPN is closely adjacent to PB2, and therefore is technically easy to be localized and isolated. More importantly, PPN isolation can avoid the use of cytoskeleton inhibitors, such as $\mathrm{CB}$ and nocodazole, which is necessary for MST and PNT. Third, PPNT manipulation is performed after fertilization, thus avoiding the premature activation of oocytes that is frequently happened in MST. We showed that PPNT resulted in a high rate of preimplantation development of reconstructed embryos even when immature MI oocytes were used. The developmental efficiency of PPNT embryos was comparable to non-manipulated ICSI controls, indicating that PPNT does not affect the developmental potential of reconstructed embryos. Of note, the reconstructed embryos obtained via PPNT in our study showed a relatively lower rate of blastocyst formation compared with that reported by studies using MST or PNT $[9,10]$ (Supplementary information, Table S1A). One potential reason could be that the MI oocytes used in our study have a lower developmental potential than mature MII oocytes used in other studies. Moreover, our study showed that the mtDNA carryover level of PPNT blastocysts and ESCs was low. Importantly, the mtDNA carryover did not increase obviously during long-term proliferation and differentiation.

In summary, we have demonstrated the technical feasibility of PPNT as a potential MRT method to prevent mtDNA disease inheritance in humans. The next challenge is to investigate the safety and efficacy of PPNT in animal models.

\section{Acknowledgments}

We thank Hongchang Li for performing PGS, Jingye Zhang and Wenrong Tao for derivation of ESCs, and Ming Gao and Yang Zou for sample preparation. We thank all participants for their support in gamete preparation. This study was supported by the National Natural Science Foundation of China (81430029, 81490743, $81622021,81601256,31371453,31571548$ and 31530048), the National Key Research and Development Program of China (2016YFC1000600), the Ministry of Science and Technology of China (2014CB964803 and 2015AA020307) and the Postdoctoral Innovation Support Program (BX201600108).

Keliang $\mathrm{Wu}^{1,2,3,4,5, *}$, Tailai Chen ${ }^{1,2,3, *}$, Sexin Huang ${ }^{1,2,3}$, Cuiqing Zhong ${ }^{4,5}$, Junhao Yan ${ }^{1,2,3}$, Xiaoyu Zhang ${ }^{6,7}$, Jinsong Li ${ }^{6,7}$, Yuan Gao ${ }^{1,2,3}$, Han Zhao ${ }^{1,2,3}$, Zi-Jiang Chen ${ }^{1,2,3,4,5}$

${ }^{I}$ Center for Reproductive Medicine, Shandong Provincial Hospital Affiliated to Shandong University, Jinan, Shandong 250001, China;

${ }^{2}$ National Research Center for Assisted Reproductive Technology and Reproductive Genetics, Jinan, Shandong 250001, China; ${ }^{3}$ The Key laboratory for Reproductive Endocrinology of Ministry of Education, Jinan, Shandong 250001, China; ${ }^{4}$ Center for Reproductive Medicine, Ren 
Ji Hospital, School of Medicine, Shanghai Jiao Tong University, Shanghai 200135, China; ${ }^{5}$ Shanghai Key Laboratory for Assisted Reproduction and Reproductive Genetics, Shanghai 200135, China; ${ }^{6}$ State Key Laboratory of Cell Biology, CAS Center for Excellence in Molecular Cell Science, Institute of Biochemistry and Cell Biology, Shanghai Institutes for Biological Sciences, Chinese Academy of Science, Shanghai 200031, China; ${ }^{7}$ Shanghai Key Laboratory of Molecular Andrology, Institute of Biochemistry and Cell Biology, Shanghai Institutes for Biological Sciences, Chinese Academy of Science, Shanghai 200031, China

*These two authors contributed equally to this work.

Correspondence: Zi-Jiang Chen ${ }^{\mathrm{a}}$, Han Zhao ${ }^{\mathrm{b}}$, Yuan Gao

${ }^{\mathrm{a}}$ Tel: +86-531-85651190

E-mail: chenzijiang@hotmail.com

${ }^{b}$ E-mail: hanzh80@yahoo.com

'E-mail: gaoyuan@sduivf.com

\section{References}

1 Wallace DC. Annu Rev Biochem 2007; 76:781-821.

2 Tachibana M, Sparman M, Sritanaudomchai H, et al. Nature 2009; 461:367-372.

3 Tachibana M, Amato P, Sparman M, et al. Nature 2013; 493:627-631.

4 Craven L, Tuppen HA, Greggains GD, et al. Nature 2010; 465:82-85.

5 Ma H, O’Neil R C, Gutierrez N M, et al. Cell Stem Cell 2017; 20:1-8.

6 Harton G L, Munné S, Surrey M, et al. Fertil Steril 2013;100:16951703.

7 Pinheiro LB, Coleman VA, Hindson CM, et al. Anal Chem 2012; 84:1003-1011.

8 Sagi I, Chia G, Golan-Lev T, et al. Nature 2016; 532:107-111.

9 Paull D, Emmanuele V, Weiss KA, et al. Nature 2013; 493:632-637.

10 Hyslop L A, Blakeley P, Craven L, et al. Nature 2016;534:383-386.

(Supplementary information is linked to the online version of the paper on the Cell Research website.) 